Gynäkologe 2011 · 44:786-787

DOI 10.1007/s00129-011-2801-z

Online publiziert: 25. September 2011

(c) Springer-Verlag 2011

\author{
M. P. Lux ${ }^{1} \cdot$ W. Jonat ${ }^{2} \cdot$ M. W. Beckmann ${ }^{1}$ \\ ${ }^{1}$ Frauenklinik, Universitätsklinikum Erlangen \\ ${ }^{2}$ Klinik für Gynäkologie und Geburtshilfe, UKSH, Campus Kiel
}

\title{
Ökonomische Rahmenbedingungen der Frauenheilkunde
}

Sehr geehrte Leserinnen und Leser,

die vorliegende Ausgabe von Der Gynäkologe beschäftigt sich mit einem ganz zentralen Thema, den ökonomischen Aspekten in unserem Fachgebiet. Die Gründe dafür, diesem Thema eine ganze Ausgaben zu widmen, liegen auf der Hand: Wir müssen uns mit den ökonomischen Aspekten auseinandersetzen, bevor diese uns unvorbereitet überrennen. Nur wer die Problematik und die dazugehörigen Daten kennt, kann gezielt Lösungen erarbeiten und konstruktiv der Zukunft begegnen.

Hintergründe sind die seit vielen Jahren steigenden Ausgaben des deutschen Gesundheitssystems, welche mittlerweile insgesamt bei mehr als 263,2 Mrd. EUR liegen. Ambulanten Einrichtungen, wie beispielsweise Arztpraxen und Apotheken, kommt hierbei mit einem Anteil von 49,7\% bzw. 130,9 Mrd. die größte Bedeutung zu. Auf die deutschen Krankenhäuser entfallen allerdings von allen einzeln betrachteten Einrichtungen mit einem Anteil von über 25\% bzw. 66,7 Mrd. EUR die größten Kosten.

Als Hauptgründe für die Zunahme der Kosten werden insbesondere die demographische Entwicklung, die Arbeitslosigkeit, der medizinisch-technische Fortschritt und das steigende Bedürfnis der Patienten nach Wohlbefinden und qualitativ hochwertigen Behandlungen (Anspruchshaltung) angesehen.

Die Folgen sind vielfältig, und das deutsche Gesundheitssystem befindet sich schon seit einigen Jahren in einem tief greifenden Struktur- und Kulturwandel. Durch den Gesetzgeber entstehen zunehmend neue Rahmenbedingungen, sie sollen zu mehr Transparenz der medizinischen Leistungen sowie der Kostenund Erlösstruktur, zu gesicherter Qualität durch kontinuierliches Qualitätsmanagement und verstärktem Streben nach Wirtschaftlichkeit der Arztpraxen und Krankenhäuser führen. Gleichzeitig sehen sich diese auch einem immer größer werdenden Wettbewerbsdruck ausgesetzt, der ihre Existenz und damit die breit ausgelegte regionale Versorgung Deutschlands bedroht. Im stationären Bereich macht vor allem der Wandel der Krankenhausfinanzierung hin zu einer von der Verweildauer weitestgehend unabhängigen Vergütung durch Fallpauschalen ein Umdenken des Managements nötig. Eine Optimierung des Betriebsergebnisses ist nur durch eine Fallzahlerhöhung oder Kostensenkungen möglich. Dafür benötigen Krankenhäuser Instrumente zur Kostenkontrolle, mit deren Hilfe die Kosten gesenkt und die Effektivität gesteigert werden sollen. Krankenhäuser werden in $\mathrm{Zu}-$ kunft dazu gezwungen sein, ihr Leistungsangebot zu optimieren. In diesem Zusammenhang müssen insbesondere Behandlungsschwerpunkte definiert werden und Überlegungen erfolgen, welche Leistungen künftig in Kooperationen mit anderen Krankenhäusern angeboten werden sollen bzw. können. Dies ist auf den ambulanten Sektor übertragbar. Auch in diesem werden zahlreiche Leistungen (z. B. Pränatalmedizin, Pränataldiagnostik) nicht sachgerecht abgebildet.
Effizienzsteigerungen durch qualitätsund kostenoptimierte Versorgungskonzepte werden insbesondere für Krankenhäuser der Maximalversorgung zunehmend wichtiger. Diese müssen einerseits die Ressourcen für Hochrisikopatienten (z. B. Früh- und Neugeborene) vorhalten, andererseits auch ein breites Leistungsangebot. Aus diesem Grund, aber auch schon, um beispielsweise die gesetzlichen Vorgaben der Struktur-, Prozess- und Ergebnisqualität zu erfüllen, ist die Bildung von Zentren ein zukunftsfähiges und immer häufiger umgesetztes Konzept.

Die Situation, in der sich die Leistungserbringer im deutschen Gesundheitswesen befinden, kann charakterisiert werden mit einem Diktum von Charles Darwin: „survival of the fittest“. (Seine Bedeutung kann mit „Überleben des am besten Angepassten" wiedergegeben werden und nicht, wie fälschlicherweise häufig angenommen, mit „Überleben des Stärksten“.) Heutzutage wird von Leistungserbringern - wie noch nie zuvor - gefordert, ihre Existenz durch ständige Anpassung an die vielschichtigen Herausforderungen langfristig zu sichern.

Ziel dieser Ausgabe ist es einerseits, die finanziellen Probleme der unterschiedlichen Bereiche unseres Fachgebietes zu beleuchten, andererseits, wesentliche gesundheitsökonomische Aspekte einschließlich der Kosteneffektivität betrachtet aus der Sicht der Kostenträger wie der Leistungserbringer - darzustellen. Kann bei zunehmenden Kosten gleichzeitig eine Kosteneffektivität der jeweiligen Leistungen und Strukturen aufgezeigt 
werden, so kann dies gezielt für Verhandlungen eingesetzt werden.

Über finanzielle Aspekte wird häufig und viel diskutiert - nicht nur konstruktiv, sondern oft auch destruktiv im Sinne einer „Untergangsstimmung“. Die vorliegende Ausgabe soll neben der Bestandsaufnahme auch eine zitierfähige Datenlage schaffen, um künftig fundiert Probleme anzugehen, Lösungen zu finden und auf Basis von publizierten Daten gezielt mit den Kostenträgern verhandeln zu können. Sowohl die Kostenträger als auch die Gesundheitspolitik sind neben Ihnen mit der aktuellen Ausgabe von Der Gynäkologe ausdrücklich angesprochen!

\section{Erlangen/Kiel, August 2011}

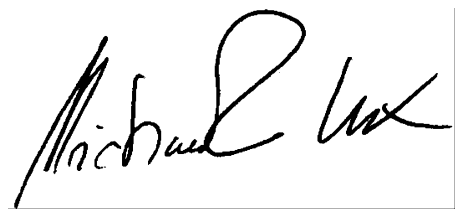

PD Dr. M. P. Lux

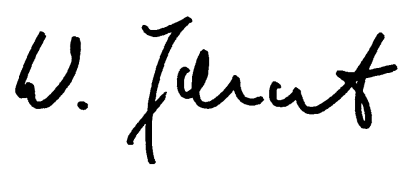

Prof. Dr. W. Jonat

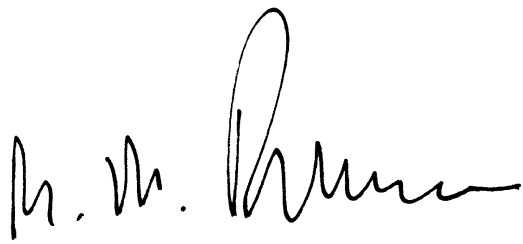

Prof. Dr. M. W. Beckmann

\section{Korrespondenzadresse}

\section{PD Dr. M. P. Lux}

Frauenklinik, Universitätsklinikum Erlangen Universitätsstr. 21-23, 91054 Erlangen michael.lux@uk-erlangen.de
Der Gynäkologe bietet jeden Monat umfassende und aktuelle Beiträge zu interessanten Themenschwerpunkten aus allen Bereichen der Gynäkologie und Geburtshilfe.

Möchten Sie ein bereits erschienenes Heft nachbestellen? Die bereits erschienenen Ausgaben aus 2010 und 2011 können Sie direkt bei unserem Kundenservice zum Preis von EUR 35,- beziehen:

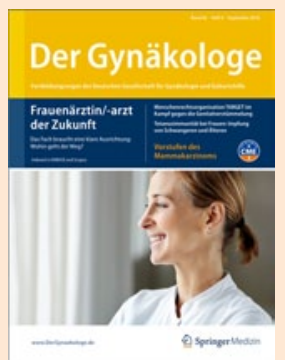

\section{Leitthemen in Der Gynäkologe 2010}

- 01/10 Verhalten in kritischen Rechtssituationen, Teil II

- 02/10 Innovationen in der minimal-invasiven Chirurgie der Gynäkologie

- 03/10 Zentrumsbildung in der Gynäkologie und Geburtshilfe

- 04/10 Hormontherapie in der Peri- und Postmenopause

- 05/10 Dokumentationssysteme in der Frauenheilkunde

- 06/10 Medizin am Rand der Lebensfähigkeit

- 07/10 Molekularbiologische Mechanismen für den Kliniker

- 08/10 Grenzsituationen in der Urogynäkologie

- 09/10 Frauenärztin/Frauenarzt der Zukunft

- 10/10 Psychosoziale Aspekte der Gynäkologie

- 11/10 Endometriose

- 12/10 Mammakarzinom - Neue Aspekte aus Praxis und Politik

\section{Leitthemen in Der Gynäkologe 2011}

- 01/11 Kontrazeption

- 02/11 Möglichkeiten und Grenzen der Reproduktionsmedizin

- 03/11 Die gynäkologisch-geburtshilfliche OP-Einheit

- 04/11 Genetik

- 05/11 Rechtliche Auflagen in Klinik und Praxis

- 06/11 Frauenärztlich-pädiatrische Kooperationen I

- 07/11 Gerinnungsstörungen in der Geburtshilfe

- 08/11 Virusinfektionen der Schwangeren

- 09/11 Update Gyn Onko: Ovar, Vulva, Endometrium

- 10/11 Ökonomische Rahmenbedingungen der Frauenheilkunde

- 11/11 Neurologische (fetale) Erkrankungen und Schwangerschaft

- 12/11 Prävention und Betreuung der übergewichtigen Patientin

(Änderungen vorbehalten)

So erreichen Sie unseren Kundenservice:

Springer Medizin Verlag GmbH

Kundenservice Zeitschriften

Haberstraße 7

69126 Heidelberg

Tel. +496221 345-4303

Fax: +49 $6221345-4229$

E-Mail: Leserservice@springer.com

www.DerGynaekologe.de 